\title{
A NEW THEORY OF PLASTIC FLOW*
}

\author{
BY \\ D. TRIFAN \\ Brown University
}

1. Introduction. This paper is concerned with a new mathematical theory of plastic flow for an isotropic, incompressible strain-hardening material exhibiting a gradual transition from the elastic to the plastic state. The discussion is restricted to states of stress and strain which can be reached by a single loading, followed by at most one partial or complete unloading.

The new stress-strain relations are introduced in Sec. 2, and the criteria for loading and unloading are established. The boundary value problem for the strain rates corresponding to given surface velocities is formulated in Sec. 3 and uniqueness of solution is proved. Sections 4 and 5 are concerned with the minimum principle associated with this boundary value problem. Sections 6 and 7 bring a comparison between the new theory and the more familiar theory of plastic deformation. ${ }^{1}$ Finally, the conditions under which the two theories furnish identical results are investigated in Sec. 8 .

2. Stress-strain relation. The customary form of the stress-strain relation of the flow theory for strain-hardening materials [2, Eq. 27] gives the rate of change of the strain tensor as a function of the stress tensor, the rate of change of the stress tensor, and the stress invariants. The treatment of some problems of practical interest is greatly facilitated, however, by the use of stress-strain relations giving the rate of change of the stress tensor explicitly in terms of the strain tensor, the rate of change of the strain tensor, and the strain invariants. Equations of this form cannot be obtained, as a rule, from the customary stress-strain relations of the flow theory. We thus are led to consider the following stress-strain relation for an incompressible material: ${ }^{2}$

$$
s_{i j}^{*}=2 G_{0} \epsilon_{i j}^{*}-p(E) E^{*} \epsilon_{i j} .
$$

Here the stress deviation $s_{i i}$ is defined as follows in terms of the stress tensor $\sigma_{i j}$ :

$$
s_{i j}=\sigma_{i j}-\frac{1}{3} \delta_{i j} \sigma_{k k} .
$$

As usual, repeated indices indicate summation in accordance with the summation convention of tensor calculus; $\delta_{i j}$ is the Kronecker delta, $\epsilon_{i i}$ is the strain tensor, $E=$ $2 \epsilon_{i j} \epsilon_{i j} / 3$ the strain intensity, $G_{0}$ the shear modulus in the elastic range, and $p=p(E)$ an arbitrary function depending on the material. Variables with asterisks indicate the rates of change of these variables with respect to a parameter $t$ which may be thought of as time. ${ }^{3}$ Stress-strain relations of the type (2.1) may be called stress theories of plastic

*Received Aug. 13, 1948. The results presented in this paper were obtained in the course of research conducted under contract sponsored jointly by the Office of Naval Research and the Bureau of Ships.

${ }^{1}$ This terminology was introduced by A. A. Ilyushin [1]. (Numbers in square brackets refer to the bibliography at the end of the paper).

2The author is indebted to W. Prager for the form of this relation which was introduced in [3].

sSince Eq. (2.1) is homogeneous in the rates of change, no viscosity effects are introduced, and the equation remains unchanged if $t$ is replaced by any monotonically increasing function $t$, i.e. by any other parameter which increases with increasing $t$. 
flow in contrast to strain theories of plastic flow such as are treated in [2, Sec. 2]. Equation (2.1) is to apply for "loading" only. The precise meaning of this term for states of combined stress will be given later.

The arbitrary function $p(E)$ in Eq. (2.1) can be determined for a particular material from the stress-strain diagram in simple tension. Denoting the tensile stress by $\sigma$ and the corresponding unit extension by $\epsilon$, the results of such a test for the type of material being considered here is given in Fig. 1. The stress-strain relation (2.1) reduces to the single equation

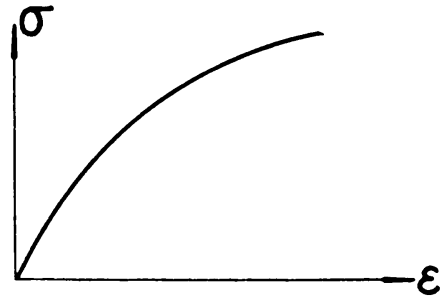

FIG. 1

$$
p(E)=\left(G_{0}-\frac{1}{3} \frac{d \sigma}{d \epsilon}\right) / E,
$$

while the strain intensity becomes $E=\epsilon^{2}$. Assuming that $\sigma(\epsilon)$ can be represented with sufficient accuracy by the power series

$$
\sigma=a_{1} \epsilon+a_{3} \epsilon^{3}+a_{5} \epsilon^{5}+\cdots,
$$

where $a_{1}=3 G_{0}$, and $a_{3}, a_{5}, \cdots$ are constants, it follows from Eqs. (2.2) and (2.3) that $p(E)$ is given by

$$
p(E)=b_{1}+b_{2} E+b_{3} E^{2}+\cdots,
$$

where $b_{n}=-(2 n+1) a_{2 n+1} / 3, n=1,2,3, \cdots$. The stress-strain relation (2.1) for loading then becomes

$$
s_{i j}^{*}=2 G_{0} \epsilon_{i j}^{*}-\left[b_{1}+b_{2} E+b_{3} E^{2}+\cdots\right] E^{*} \epsilon_{i i} .
$$

The stress-strain relation for unloading will be assumed to be given by the differentiated form of Hooke's law:

$$
s_{i j}^{*}=2 G_{0} \epsilon_{i j}^{*} .
$$

Under combined states of stress and strain, loading may occur in certain portions of a body and unloading in others, so that it becomes necessary to establish some sort of loading-unloading criterion. For that purpose we will assume that loading takes place wherever $\epsilon_{i j} d \sigma_{i j}>0$, and unloading wherever $\epsilon_{i j} d \sigma_{i j}<0$. If we multiply Eq. (2.1) by the strain tensor, we find that

$$
\sigma_{i j}^{*} \epsilon_{i j}=\frac{3}{2} E^{*}\left(G_{0}-E p(E)\right)>0
$$

for loading. On the other hand, all along the stress-strain curve of Fig. 1 the slope satisfies

$$
0<\frac{d \sigma}{d \epsilon} \leq 3 G_{0},
$$


and therefore, by Eq. (2.2),

$$
0 \leq p(E)<\frac{G_{0}}{E}
$$

It follows from Eq. (2.7) and (2.8) that $E^{*}>0$ for loading. A similar procedure with Eq. (2.6) shows that the unloading criterion is $E^{*}<0$.

The stress-strain relations for loading and unloading can be combined into one expression, namely

$$
s_{i j}^{*}=2 G_{0} \epsilon_{i j}^{*}-\frac{1}{2} p(E) \epsilon_{i j}\left(E^{*}+\left|E^{*}\right|\right)
$$

which reduces to Eq. (2.1) when $E^{*}>0$ and to $(2.6)$ when $E^{*}<0$.

3. Uniqueness of solution. With the aid of two lemmas which are to be established in this section the following uniqueness theorem will be proved.

Theorem. For a given state of strain $\epsilon_{i j}$ throughout the body, and given velocities $u_{i}^{*}$ on the surface such that the surface integral of the normal velocity component vanishes, the rate of change of strain $\epsilon_{i j}^{*}$ is uniquely determined throughout the body.

Lemma 1. Let $\epsilon_{i j}$ be some fixed state of strain throughout the body, and $\epsilon_{i j}^{* \prime}$ and $\epsilon_{i j}^{* \prime \prime}$ two arbitrary sets of strain rates which satisfy the condition of incompressibility. Furthermore, let $\sigma_{i j}^{* \prime}$ and $\sigma_{i j}^{* \prime \prime}$ be the corresponding stress rates obtained from the stressstrain relation (2.9). Then

$$
\left(\epsilon_{i j}^{* \prime}-\epsilon_{i j}^{* \prime \prime}\right)\left(\sigma_{i j}^{* \prime}-\sigma_{i j}^{* \prime \prime}\right)>0
$$

holds throughout the body independent of whether there is local loading or unloading.

Proof. Setting $d_{i j}^{*}=\epsilon_{i j}^{* \prime}-\epsilon_{i j}^{* \prime \prime}$ and noting that $d_{i j}^{*}\left(\sigma_{i j}^{* \prime}-\sigma_{i j}^{* \prime \prime}\right)=d_{i j}^{*}\left(s_{i j}^{* \prime}-s_{i j}^{* \prime \prime}\right)$, we find from Eq. (2.9), that

$$
d_{i j}^{*}\left(s_{i j}^{* \prime}-s_{i j}^{* \prime \prime}\right)=2 G_{0} d_{i j}^{*} d_{i j}^{*}-\frac{1}{2} p(E) d_{i j}^{*} \epsilon_{i j}\left\{E^{* \prime}-E^{* \prime \prime}+\left|E^{* \prime}\right|-\left|E^{* \prime \prime}\right|\right\} .
$$

Because

$$
\begin{gathered}
\left|\left\{E^{* \prime}-E^{* \prime \prime}+\left|E^{* \prime}\right|-\left|E^{* \prime \prime}\right|\right\}\right| \leq\left|E^{* \prime}-E^{* \prime \prime}\right|+|| E^{* \prime}|-| E^{* \prime \prime}|| \\
\leq 2\left|E^{* \prime}-E^{* \prime \prime}\right|
\end{gathered}
$$

and

$$
E^{* \prime}-E^{* \prime \prime}=\frac{4}{3} \epsilon_{r s} d_{r s}^{*}
$$

we have

$$
d_{i j}^{*}\left(s_{i j}^{* \prime}-s_{i j}^{* \prime \prime}\right) \geq 2 G_{0} d_{i j}^{*} d_{i j}^{*}-\frac{4}{3} p(E)\left(d_{i j}^{*} \epsilon_{i j}\right)^{2}
$$

since $p(E) \geq 0$ by Eq. (2.8). By the Schwarzian inequality

$$
\left(d_{i j}^{*} \epsilon_{i j}\right)^{2} \leq d_{i j}^{*} d_{i}^{*} \epsilon_{r s} \epsilon_{r s}=\frac{3}{2} d_{i j}^{*} d_{i j}^{*} E
$$

so that

$$
d_{i j}^{*}\left(s_{i j}^{* \prime}-s_{i j}^{* \prime \prime}\right) \geq 2 d_{i j}^{*} d_{i j}^{*}\left\{G_{0}-E p(E)\right\}>0 .
$$

This proves the lemma (see Eq. (2.8)). 
Lemma 2. Let $V$ be any "regular region" [4, p. 112] with surface $S$ which is the sum of a finite number of "normal regions" [4, p. 85], $V_{1}, V_{2}, \cdots, V_{n}$, and $\sigma_{i}^{* \prime}$ and $\epsilon_{i}^{* \prime \prime}$ functions satisfying the following conditions:

1) in each normal region
a) $\sigma_{i j}^{* \prime}=\sigma_{i}^{* \prime}$
b) $\sigma_{i j}^{* \prime}$ is continuous and differentiable,
c) $\sigma_{i j, k}^{* \prime} \equiv \partial \sigma_{i j}^{* \prime} / \partial x_{k}$ is continuous,
d) $\sigma_{i, i}^{* \prime}=0$ (equation of equilibrium);

2) the rates of change of the stresses transmitted across the surface of separation between two adjacent normal regions are the same whether evaluated from $\sigma_{i j}^{* \prime}$ on one side or $\sigma_{i j}^{* \prime}$ on the other;

3 ) in each normal region

a) $\epsilon_{i j}^{* \prime \prime}$ is continuous,

b) $\epsilon_{i j}^{* \prime \prime}=1 / 2\left(u_{i, i}^{* \prime \prime}+u_{i, i}^{* \prime \prime}\right)$; and

4) the velocities $u_{i}^{* \prime \prime}$ are continuous throughout $V+S$. Then

$$
\int_{V} \sigma_{i j}^{* \prime} \epsilon_{i j}^{* \prime \prime} d v=\int_{S} \sigma_{i j}^{* \prime} u_{i}^{* \prime \prime} n_{i} d S,
$$

where $n_{j}$ is the external normal to the surface.

Proof. Using the conditions (1b), (3a), (3b), (1a), (1d), we find

$$
\int_{V} \sigma_{i j}^{* \prime} \epsilon_{i j}^{* \prime \prime} d v=\sum_{1}^{n} \int_{V_{k}} \sigma_{i j}^{* \prime} \epsilon_{i j}^{* \prime \prime} d v=\sum_{1}^{n} \int_{V_{k}}\left(\sigma_{i}^{* \prime} u_{i}^{* \prime \prime}\right)_{, j} d v .
$$

Use of conditions (1c), (2), (4), and the divergence theorem then gives us

$$
\sum_{i}^{n} \int_{V_{k}}\left(\sigma_{i j}^{* \prime} u_{i}^{* \prime \prime}\right)_{, j} d v=\int_{S} \sigma_{i j}^{* \prime} u_{i}^{* \prime \prime} n_{i} d S .
$$

It is now possible to prove the uniqueness theorem stated earlier. Let us assume that two solutions $\epsilon_{i i}^{*}$ and $\epsilon_{i j}^{* \prime}$ exist each deriving from a system of velocities in accordance with

$$
\epsilon_{i j}^{*}=\frac{1}{2}\left(u_{i, j}^{*}+u_{i, i}^{*}\right)
$$

and

$$
\epsilon_{i, j}^{* \prime}=\frac{1}{2}\left(u_{i, i}^{* \prime}+u_{i, i}^{* \prime}\right),
$$

where $u_{i}^{*}=u_{i}^{* \prime}$ on the boundary. Since $\sigma_{i j}^{*}$ and $\sigma_{i j}^{* \prime}$, the corresponding rates of change of stress obtained from Eq. (2.9), represent solutions, they must satisfy the equations of equilibrium, $\sigma_{i, j}^{*}=0$ and $\sigma_{i, j}^{* \prime}=0$. It is assumed that no body forces exist. All the conditions of Lemma 2 being satisfied, we can conclude that

$$
\int_{V}\left(\epsilon_{i j}^{*}-\epsilon_{i j}^{* \prime}\right)\left(\sigma_{i j}^{*}-\sigma_{i j}^{* \prime}\right) d v=\int_{S}\left(u_{i}^{*}-u_{i}^{* \prime}\right)\left(\sigma_{i j}^{*}-\sigma_{i j}^{* \prime}\right) n_{j} d S .
$$

But since on the surface $u_{i}^{*}=u_{i}^{* \prime}$ the surface integral vanishes and hence the volume integral also. On the other hand, by Lemma 1 the volume integral is greater than zero, unless $\epsilon_{i j}^{*}=\epsilon_{i j}^{* \prime}$. Thus, the solution $\epsilon_{i j}^{*}$ must be unique. 
4. A minimum principle for loading throughout the body. We shall confine ourselves in this section to the case in which loading takes place throughout the entire body. We denote existing strains by $\epsilon_{i j}$ and the solution, i.e., the strain rates which actually occur, by $\epsilon_{i j}^{*}$. These strain rates must satisfy the following:

1) the equation of equilibrium $\sigma_{i, j}^{*}=0$,

2) $\epsilon_{k k}^{*}=0$

3) $\epsilon_{i j}^{*}=\frac{1}{2}\left(u_{i, j}^{*}+u_{i, i}^{*}\right)$, and

4) $\epsilon_{i j} \epsilon_{i j}^{*}>0$,

where $u_{i}^{*}$ equals the prescribed boundary values on the surface $S$. Let $\epsilon_{i}^{* \prime}$ be an arbitrary set of strain rates which satisfy the conditions:

1) $\epsilon_{k k}^{* \prime}=0$,

2) $\epsilon_{i j}^{* \prime}=\frac{1}{2}\left(u_{i, j}^{* \prime}+u_{i, i}^{* \prime}\right), \quad$ and

3) $\epsilon_{i j} \epsilon_{i j}^{* \prime}>0$,

where $u_{i}^{* \prime}=u_{i}^{*}$ on the surface. With these restrictions on the natural strain rates $\epsilon_{i j}^{*}$ and the admissible strain rates $\epsilon_{i j}^{* \prime}$ the following minimum principle can be established.

Minimum Principle.

$$
\int_{V} \epsilon_{i j}^{*} \sigma_{i j}^{*} d v \leq \int_{V} \epsilon_{i j}^{* \prime} \sigma_{i j}^{* \prime} d v
$$

when $\sigma_{i j}^{*}$ and $\sigma_{i j}^{* \prime}$ are obtained from $\epsilon_{i j}^{*}$ and $\epsilon_{i j}^{* \prime}$, respectively, by means of the stressstrain relation (2.9). Equality holds if and only if $\epsilon_{i j}^{*}=\epsilon_{i j}^{* \prime}$.

In proving this principle we use the following lemma.

Lemma 3. For the stress rates and strain rates introduced above

$$
\epsilon_{i j}^{*} \sigma_{i j}^{* \prime}=\epsilon_{i j}^{* \prime} \sigma_{i j}^{*} .
$$

Proof. From $\epsilon_{i j}^{*} \sigma_{i j}^{* \prime} \equiv \epsilon_{i j}^{*} s_{i j}^{* \prime}$ and $\epsilon_{i j}^{* \prime} \sigma_{i j}^{*} \equiv \epsilon_{i j}^{* \prime} s_{i j}^{*}$, the lemma is obtained by applying Eq. (2.9) for $E^{*}>0$ and $E^{* \prime}>0$. The equality also holds when $E^{*}<0$ and $E^{* \prime}<0$. We note that Lemma 3 would not hold if, for instance, $\epsilon_{i j}^{*}$ were to constitute loading and $\epsilon_{i j}^{* \prime}$ unloading.

Returning to the minimum principle it follows from Lemma 1 that

$$
\int_{V} \epsilon_{i j}^{*} \sigma_{i j}^{*} d v-\int_{V} \epsilon_{i j}^{* \prime} \sigma_{i j}^{*} d v-\int_{V} \epsilon_{i j}^{*} \sigma_{i j}^{* \prime} d v+\int_{V} \epsilon_{i j}^{* \prime} \sigma_{i j}^{* \prime} d v \geq 0,
$$

where equality holds if and only if $\epsilon_{i j}^{*}=\epsilon_{i j}^{* \prime}$. According to Lemma 2,

$$
\int_{V} \epsilon_{i ;}^{* \prime} \sigma_{i ;}^{*} d v=\int_{S} u_{i}^{* \prime} \sigma_{i j}^{*} n_{i} d S ;
$$

and

$$
\int_{V} \epsilon_{i ;}^{*} \sigma_{i ;}^{*} d v=\int_{S} u_{i}^{*} \sigma_{i ;}^{*} n_{i} d S
$$


Since $u_{i}^{* \prime}=u_{i}^{*}$ on $S$ it follows that

$$
\int_{V} \epsilon_{i j}^{* \prime} \sigma_{i j}^{*} d v=\int_{V} \epsilon_{i j}^{*} \sigma_{i j}^{*} d v .
$$

By Lemma 3 and (4.2), we thus have

$$
\int_{V} \epsilon_{i j}^{*} \sigma_{i j}^{* \prime} d v=\int_{V} \epsilon_{i j}^{*} \sigma_{i j}^{*} d v .
$$

Substitution of relations (4.2) and (4.3) into (4.1) completes the proof.

5. Extension of the minimum principle. For the case where loading occurs in a sub-domain of the body and unloading occurs everywhere else, ${ }^{4}$ some of the arguments presented in Sec. 4 are no longer valid because Lemma 3 cannot be applied throughout the body. Since the actual surface of separation between the two domains of loading and unloading for $\epsilon_{i j}^{*}$ is not known, the surface which separates the regions of loading and unloading for $\epsilon_{i j}^{* \prime}$ is in general distinct from the actual surface. Accordingly, the domain $V$ is divided by these surfaces into what we assume to be four normal subdomains, namely $V_{+_{+}}, V_{-_{+}}, V_{+_{-}}$, and $V_{--}$; the first of the two subscripts refers to the actual state, and the second to the artificial state, the plus sign denoting loading and the negative sign unloading.

Both (4.1) and (4.2) are still valid in this more general case, so that

$$
\int_{V} \epsilon_{i j}^{*} \sigma_{i j}^{* \prime} d v \leq \int_{V} \epsilon_{i j}^{* \prime} \sigma_{i j}^{* \prime} d v .
$$

Since Lemma 3 applies over the domains $V_{++}$and $V_{--}$, we have

$$
\int_{V} \epsilon_{i j}^{*} \sigma_{i j}^{* \prime} d v=\int_{V_{++}} \epsilon_{i j}^{* \prime} \sigma_{i j}^{*} d v+\int_{V_{--}} \epsilon_{i j}^{* \prime} \sigma_{i j}^{*} d v+\int_{V_{-+}} \epsilon_{i j}^{*} \sigma_{i j}^{* \prime} d v+\int_{V_{+-}} \epsilon_{i j}^{*} \sigma_{i j}^{* \prime} d v,
$$

or

$$
\int_{V} \epsilon_{i j}^{*} \sigma_{i j}^{* \prime} d v=\int_{V} \epsilon_{i j}^{* \prime} \sigma_{i j}^{*} d v+\int_{V-+} L d v+\int_{V_{+-}} L d v
$$

where

$$
L=\epsilon_{i j}^{*} \sigma_{i j}^{* \prime}-\epsilon_{i j}^{* \prime} \sigma_{i j}^{*} \equiv \epsilon_{i j}^{*} s_{i j}^{* \prime}-\epsilon_{i j}^{* \prime} s_{i j}^{*} .
$$

By use of Eq. (2.9), we find that

$$
L=\frac{3}{8} p(E)\left\{E^{* \prime}\left|E^{*}\right|-E^{*}\left|E^{* \prime}\right|\right\} .
$$

Using Eqs. (5.2), (5.3), (4.2) and the fact that $E^{*}<0, E^{* \prime}>0$ in $V_{-+}$and $E^{*}>0$, $E^{* \prime}<0$ in $V_{+-}$, we write $(5.1)$ in the form

$$
\int_{V} \epsilon_{i j}^{*} \sigma_{i j}^{*} d v-\frac{3}{4} \int_{V-+} p(E) E^{*} E^{* \prime} d v+\frac{3}{4} \int_{V_{+-}} p(E) E^{*} E^{* \prime} d v \leq \int_{V} \epsilon_{i j}^{* \prime} \sigma_{i j}^{* \prime} d v .
$$

In the domains $V_{-+}$and $V_{+-}$, we have $p E^{*} E^{* \prime} \leq 0$ since $p(E) \geq 0$ (see (2.8)).

${ }^{4} \mathrm{It}$ is assumed here that the domains of loading and unloading separate the body into two normal regions. This condition is not necessary and is introduced for the purpose of simplification only. The generalization to any finite number of normal regions is immediate. 
We conclude that the inequality

$$
\int_{V} \epsilon_{i j}^{*} \sigma_{i j}^{*} d v \leq \int_{V} \epsilon_{i j}^{* \prime} \sigma_{i j}^{* \prime} d v
$$

holds even in this more general case, whenever the artificial state is made to constitute loading in those regions where the actual state constitutes loading. In this case there is no $V_{+-}$domain. ${ }^{5}$

6. Stress-strain relations for a theory of plastic deformation. The customary stressstrain relations [6, p. 75] for incompressible materials possessing the property of gradual transition from the elastic to the plastic state are

$$
s_{i i}=2 G^{\prime} \epsilon_{i i},
$$

where $G^{\prime}=G^{\prime}(E)$ is the secant shear modulus. The secant shear modulus, being a function of the strain intensity $E$ alone, can be evaluated for any material in the manner in which the quantity $p(E)$ was obtained for the flow theory.

For simple tension Eq. (6.1) reduces to the single expression

$$
\sigma / \epsilon=3 G^{\prime}
$$

Using the same series expansion (2.3) as in the plastic flow theory, we obtain

$$
3 G^{\prime}=3 G_{0}+a_{3} E+a_{5} E^{2}+\cdots .
$$

Substituting this into Eq. (6.1) we find

$$
s_{i i}=2 G_{0} \epsilon_{i i}-\left[c_{1}+c_{2} E+c_{3} E^{2}+\cdots\right] E \epsilon_{i i},
$$

where $c_{n}=-2 a_{2 n+1} / 3$ and $n=1,2,3, \cdots$.

7. Comparison between the two plastic theories. Consider a solid composed of a strain-hardening material which is deformed from a state of zero stress and strain by an equilibrium system of surface stresses such that loading occurs throughout the body. We assume that the relation between stress and strain in simple tension for the material, as given by the series expansion

$$
\sigma=a_{1} \epsilon+a_{3} \epsilon^{3}+a_{5} \epsilon^{5}+\cdots,
$$

is known. The complete systems of equations for the two theories are then as follows.

I. Plastic Flow Theory.

a) Equations of equilibrium

$$
\sigma_{i, i}^{*}=0,
$$

where body forces are neglected;

b) condition of incompressibility

$$
\epsilon_{k k}^{*}=0 ;
$$

c) stress-strain relation

$$
s_{i j}^{*}=2 G_{0} \epsilon_{i i}^{*}-\left[b_{1}+b_{2} E+b_{3} E^{2}+\cdots\right] E^{*} \epsilon_{i i},
$$

where $b_{n}=-(2 n+1) a_{2 n+1} / 3$; 
d) strain rate-velocity relation

$$
\epsilon_{i j}^{*}=\frac{1}{2}\left(u_{i, i}^{*}+u_{i, i}^{*}\right),
$$

where the velocity vector $u_{i}^{*}$ is assumed small; and

e) boundary conditions

$$
u_{i}^{*}=U_{i}^{*},
$$

where $U_{i}^{*}$ is the prescribed velocity of the surface.

Equations $I(a)-I(d)$ must be satisfied at each interior point of the solid, while $I(e)$ must hold on the surface.

II. Plastic Deformation Theory.

a) $\sigma_{i, i}=0$

b) $\epsilon_{k k}=0$;

c) $s_{i j}=2 G_{0} \epsilon_{i j}-\left[c_{1}+c_{2} E+c_{3} E^{2}+\cdots\right] E \epsilon_{i j}, \quad$ where $c_{n}=-2 a_{2 n+1} / 3$;

d) $\epsilon_{i j}=\frac{1}{2}\left(u_{i, j}+u_{i, i}\right) ; \quad$ and

e) $u_{i}=U_{i}$.

The possibility of solving these non-linear partial differential equations for $\sigma_{i j}$ and $u_{i}$ in closed form is remote for most practically important problems. The procedure to be used in this paper assumes that the solutions may be represented by power series expansions of the form

$$
\begin{aligned}
& \sigma_{i j}=\sum_{p=0}^{\infty} \sigma_{i j}^{(2 p+1)} t^{2 p+1}, \\
& \epsilon_{i j}=\sum_{p=0}^{\infty} \epsilon_{i j}^{(2 p+1)} t^{2 p+1} \\
& u_{i}=\sum_{p=0}^{\infty} u_{i}^{(2 p+1)} t^{2 p+1},
\end{aligned}
$$

and

$$
U_{i}=\sum_{\nu=0}^{\infty} U_{i}^{(2 \nu+1)} t^{2 p+1},
$$

where the unknown coefficients of the odd powers of the conveniently chosen parameter $t$, which may be thought of as time, depend on the space variables only. Substituted in the basic equations of the flow theory these expansions lead to the following relations:
a) $\sigma_{i j, i}^{(1)}+3 \sigma_{i j, i}^{(3)} t^{2}+5 \sigma_{i j, i}^{(5)} t^{4}+\cdots=0$;
b) $\epsilon_{k k}^{(1)}+3 \epsilon_{k k}^{(3)} t^{2}+5 \epsilon_{k k}^{(5)} t^{4}+\cdots=0$;
c) $s_{i j}^{(1)}+3 s_{i j}^{(3)} t^{2}+5 s_{i j}^{(5)} t^{4}+\cdots=2 G_{0}\left\{\epsilon_{i j}^{(1)}+3 \epsilon_{i j}^{(3)} t^{2}+5 \epsilon_{i j}^{(5)} t^{4}+\cdots\right\}$ 


$$
\begin{aligned}
& -\frac{4}{3}\left[b_{1}+\frac{2}{3} b_{2}\left(E_{11} t^{2}+2 E_{13} t^{4}+\cdots\right)\right. \\
& \left.+\frac{4}{9} b_{3}\left(E_{11}^{2} t^{4}+4 E_{11} E_{13} t^{6}+\cdots\right)+\cdots\right] \\
& \cdot\left[E_{11} t+4 E_{13} t^{3}+\cdots\right]\left[\epsilon_{i j}^{(1)} t+\epsilon_{i j}^{(3)} t^{3}+\epsilon_{i j}^{(5)} t^{5}+\cdots\right],
\end{aligned}
$$

where $E_{r s}=\epsilon_{i j}^{(r)} \epsilon_{i j}^{(s)}$

$$
\text { d) } \begin{aligned}
\epsilon_{i j}^{(1)} & +3 \epsilon_{i j}^{(3)} t^{2}+5 \epsilon_{i j}^{(5)} t^{4}+\cdots=\frac{1}{2}\left[\left\{u_{i, j}^{(1)}+3 u_{i, j}^{(3)} t^{2}+5 u_{i, j}^{(5)} t^{4}+\cdots\right\}\right. \\
& \left.+\left\{u_{j, i}^{(1)}+3 u_{i, i}^{(3)} t^{2}+5 u_{i, i}^{(5)} t^{4}+\cdots\right\}\right]
\end{aligned}
$$

and
e) $u_{i}^{(1)}+3 u_{i}^{(3)} t^{2}+5 u_{i}^{(5)} t^{4}+\cdots=U_{i}^{(1)}+3 U_{i}^{(3)} t^{2}+5 U_{i}^{(5)} t^{4}+\cdots$.

The unknown coefficients of the linear terms in $t$ of the expansions (7.1) are the solutions of the set of differential equations obtained for $t=0$ in Eqs. (7.2), namely
a) $\sigma_{i j, i}^{(1)}=0$
b) $\epsilon_{k k}^{(1)}=0$;
c) $s_{i j}^{(1)}=2 G_{0} \epsilon_{i j}^{(1)}$
d) $\epsilon_{i j}^{(1)}=\frac{1}{2}\left\{u_{i, j}^{(1)}+u_{i, i}^{(1)}\right\} ; \quad$ and
e) $u_{i}^{(1)}=U_{i}^{(1)}$.

These equations represent the basic equations for an incompressible elastic body, for which the existence $[7,8]$ and uniqueness $[9$, pp. 92, 93] are known. We assume that the linear equations (7.3) can then be integrated either in closed form or by some numerical method to yield $\sigma_{i j}^{(1)}$ and $u_{i}^{(1)}$. Substituting Eqs. (7.3) into (7.2), factoring out $t^{2}$, and letting $t$ tend towards zero, we obtain
a) $\sigma_{i i, i}^{(3)}=0 ;$
b) $\epsilon_{k k}^{(3)}=0$;
c) $s_{i j}^{(3)}=2 G_{0} \epsilon_{i j}^{(3)}-\frac{4}{9} b_{1} E_{11} \epsilon_{i j}^{(1)}$
d) $\epsilon_{i j}^{(3)}=\frac{1}{2}\left\{u_{i, j}^{(3)}+\dot{u}_{i, i}^{(3)}\right\} ; \quad$ and
e) $u_{i}^{(3)}=U_{i}^{(3)}$.

These equations, too, have the form of the basic equations for an incompressible elastic body. Indeed, they are equivalent to 

a) $\sigma_{i j, i}^{(3)}=\frac{4}{9} b_{1}\left(E_{11} \epsilon_{i j}^{(1)}\right)_{, i}$;
b) $\epsilon_{k k}^{(3)}=0$;
c) $s_{i j}^{(3)}=2 G_{0} \epsilon_{i j}^{(3)}$
d) $\epsilon_{i j}^{(3)}=\frac{1}{2}\left\{u_{i, j}^{(3)}+u_{i, i}^{(3)}\right\} ; \quad$ and
e) $u_{i}^{(3)}=U_{i}^{(3)}$,

where we introduce a fictitious body force expressed in terms of the solution of the first system of Eqs. (7.3). It is readily seen that all other unknown coefficients in the expansions (7.1) can be calculated from sets of equations similar in structure to Eqs. (7.5).

Carrying out an analogous procedure in the case of the basic equations of the plastic deformation theory we find that the calculation of the unknown coefficients of the linear terms in $t$ of expansions (7.1) reduces, as for the flow theory, to the solving of Eqs. (7.3). The differential equations for $\sigma_{i j}^{(3)}, \epsilon_{i j}^{(3)}$, etc. are those of Eqs. (7.4) with the exception that the factor $4 b_{1} / 9$ in Eq. (7.4c) is replaced by the factor $2 c_{1} / 3$; however, from the definitions,

$$
\frac{4}{9} b_{1}=\frac{2}{3} c_{1}=-\frac{4}{9} a_{3}
$$

We thus find that the two theories give solutions which are identical as far as the terms. linear and cubic in $t$ are concerned.

The next higher order approximation will produce a difference between the solutions. as given by the flow and deformation theories. For the flow theory, we have

$$
s_{i j}^{(5)}=2 G_{0} \epsilon_{i j}^{(5)}+\frac{4}{15} a_{3} E_{11} \epsilon_{i j}^{(3)}+\frac{16}{15} a_{3} E_{13} \epsilon_{i j}^{(1)}+\frac{8}{27} a_{5} E_{11}^{2} \epsilon_{i j}^{(1)},
$$

and for the deformation theory

$$
s_{i j}^{(5)}=2 G_{0} \epsilon_{i j}^{(5)}+\frac{4}{9} a_{3} E_{11} \epsilon_{i j}^{(3)}+\frac{8}{9} a_{3} E_{13} \epsilon_{i j}^{(1)}+\frac{8}{27} a_{5} E_{11}^{2} \epsilon_{i j}^{(1)} .
$$

Thus, from this stage on, the two theories will differ as a rule.

8. Conditions for similarity. In order that the fifth order terms be the same for both theories, so that the solutions agree up to the terms of fifth order, we have the condition

$$
E_{13} \epsilon_{i j}^{(1)}=E_{11} \epsilon_{i j}^{(3)}
$$

This equation is satisfied whenever

$$
\epsilon_{i j}^{(3)}=f(x, y, z) \epsilon_{i j}^{(1)},
$$

where $f(x, y, z)$ is an arbitrary function. However, the strains $\epsilon_{i j}^{(3)}$ as given by (8.2) will satisfy the compatibility equations [9, p. 27] only if the function $f(x, y, z)$ reduces to a constant. Accordingly,

$$
\epsilon_{i j}^{(3)}=A \epsilon_{i j}^{(1)}
$$


If we define $\epsilon_{i j} / E^{1 / 2}$ as the normalized tensor of $\epsilon_{i j}$, condition (8.3) states that the normalized tensors $\epsilon_{i j}^{(1)}$ and $\epsilon_{i j}^{(3)}$ must be identical. Substituting this value of $\epsilon_{i j}^{(3)}$ into the stress-strain relations for $s_{i j}^{(7)}$ and adding a further restriction that

$$
\epsilon_{i j}^{(5)}=B \epsilon_{i j}^{(1)}
$$

we find that now the two theories are identical for seventh order terms. Thus the first four terms of the expansion for $s_{i j}$, i.e.,

$$
s_{i j}=s_{i j}^{(1)} t+s_{i j}^{(3)} t^{3}+s_{i j}^{(5)} t^{5}+s_{i j}^{(7)} t^{7}
$$

are identical for both theories whenever

$$
\epsilon_{i j}=\epsilon_{i j}^{(1)}\left[t+A t^{3}+B t^{5}\right]+\epsilon_{i j}^{(7)} t^{7} .
$$

Higher order agreement may be obtained by similar restrictions.

It is of interest to determine conditions under which the actual solutions as given by the theories of plastic flow and plastic deformation, and not as approximated by a finite number of terms of expansions (7.1), are identical. Differentiating the plastic deformation equations II with respect to time and comparing with the plastic flow equations I, we find that there is no difference between the theories if

$$
2 E \epsilon_{i j}^{*}-E^{*} \epsilon_{i j}=0 .
$$

This condition is fulfilled if

$$
\epsilon_{i i}=\epsilon_{i j}^{(1)}(x, y, z) g(t),
$$

where $g(t)$ is a monotonically increasing function of $t$. The two theories thus are seen to furnish identical predictions if the ratios between the various strain components at a generic point remain constant throughout the loading process.

\section{BibLIOGRAPHY}

1. A. A. Ilyushin, Relation between the theory of Saint Venant-Lévy-Mises and the theory of small elastic-plastic deformations, Prikl. Mat. Mekh. 9, 207-218 (1945).

2. G. H. Handelman, C. C. Lin, and W. Prager, On the mechanical behavior of metals in the strainhardening range, Q. Appl. Math. 4, 397-407 (1947).

3. D. Trifan, On the plastic bending of circular plates under uniform transverse loads, Q. Appl. Math. 6, 417-427 (1948).

4. O. D. Kellogg, Foundations of potential theory, Murray Printing Co., New York, 1929.

5. P. Hodge and W. Prager, A variational principle for plastic materials with strain-hardening, J. Math. Phys. 27, 1-10 (1948).

6. A. Nadai, Plasticity, a mechanics of the plastic state of matter, McGraw-Hill Book Co., Inc., New York, 1931.

7. A. Korn, Über die Lösung des Grundproblems der Elastizitätstheorie, Math. Ann. 75, 497-544 (1914). (1924).

8. L. Lichtenstein, Über die erste Randwertaufgabe der Elastizitätstheorie, Math. Zeit. 20, 21-28

9. I. S. Sokolnikoff, Mathematical theory of elasticity, McGraw-Hill Book Co., Inc., New York, 1946. 